\title{
Identification of Potential core genes in Sevoflurance induced Myocardial Energy Metabolism in Patients Undergoing Off-pump Coronary Artery Bypass Graft Surgery using Bioinformatics analysis
}

Hua Lin ( $\nabla$ nmghlelh@sina.com )

Tianjin Medical University General Hospital Airport Site

Research article

Keywords: sevoflurane, Myocardial Energy Metabolism, Off-pump Coronary Artery Bypass Graft Surgery

Posted Date: November 18th, 2019

DOI: https://doi.org/10.21203/rs.2.17434/v1

License: (c) (i) This work is licensed under a Creative Commons Attribution 4.0 International License. Read Full License 


\section{Abstract}

Background: Myocardial ischemia-reperfusion injury always happened after Off-pump coronary artery bypass graft(OPCABG), and this can not be avoided altogether. In this study, we tried to detect potential genes of sevoflurane-induced myocardial energy metabolism in patients undergoing OPCABG using bioinformatics analysis.

Methods: We download and analyze the gene expression profile data from the Gene Expression Omnibus(GEO) database using bioinformatics methods. We downloded the gene expression data from the Gene Expression Omnibus(GEO) database using bioinformatics methods. Gene Ontology(GO) functional annotation analysis and Kyoto Encyclopedia of Genes and Genomes(KEGG) pathway enrichment analysis were used to analysis the screened differentially expressed genes(DEGs). Then, we established a protein-protein interaction (PPI) network to find hub genes associated with myocardial energy metabolism.

Results: Through PPI network, we find ten hub genes, including JUN, EGR1, ATF3, FOSB, JUNB, DUSP1, EGR2, NR4A1, BTG2, NR4A2.

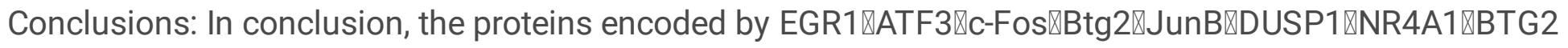
and NR4A2 were related to cardiac function. ATF3, FOSB, JUNB, DUSP1, NR4A1, NR4A2 are related to apoptosis of cardiomyocytes. The protein encoded by BTG2 is related to hypertrophy.

Sevoflurane regulates cell transcription, inflammatory and apoptosis through those hub genes to protect myocardial.

\section{Background}

Off-pump coronary artery bypass graft(OPCABG) surgery is an effective way to avoid the side effect of extracorporeal circulation, like the whole-body inflammatory syndrome, Postoperative cognitive dysfunction, coagulation disorders, and multiple organ dysfunction syndromes. At present, gas anaesthesia, sevoflurane has been widely used during the CABG.1 Coronary artery bypass grafting(CABG) is an effective way to treat left primary coronary disease or three-vessel disease. Lousy lifestyle and habits cause the happening of coronary artery disease(CAD) in China increased gradually. Patients with clinical symptoms and multiple CAD need surgery. Off-pump coronary artery bypass graft(OPCABG) is a right choice of surgical procedure. Even so, after OPCABG, myocardial ischemia-reperfusion injury can also not be avoided entirely and happened high probability. ${ }^{3,5}$ In this study, we tried to detect potential genes of sevoflurane-induced myocardial energy metabolism in patients undergoing OPCABG. To find the differentially expressed genes (DEGs) between before inhalation (baseline sevoflurane) and after inhalation (sevoflurane), We download and analyze the gene expression profile data from the Gene Expression Omnibus(GEO) database using bioinformatics methods. Gene Ontology(GO) functional annotation analysis and Kyoto Encyclopedia of Genes and Genomes(KEGG) pathway enrichment 
analysis were performed for the screened DEGs. Then, we established a protein-protein interaction (PPI) network to find hub genes related to myocardial energy metabolism.

Functional analysis of DEGs was selected using Gene Ontology(GO) database and signal pathway of DEGs was carried out using the Kyoto Encyclopedia of Genes and Genomes(KEGG). Then, through the search tools, protein-protein interaction(PPI) network and hub genes related to myocardial energy metabolism were selected.

\section{Methods}

\section{Materials and methods}

We download a database from the GEO database(https://www.ncbi.nlm.nih. gov/geo/) to obtain the gene expression datasets. One series(GDS2772) was selected out from the database about sevoflurane affect human myocardial energy metabolism. GDS2772 was based on the Agilent GPL570: [HG-U133_Plus_2] Affymetrix Human Genome U133 Plus 2.0 Array. This data was available online searched by "sevoflurane" and "myocardial energy metabolism". This study has not been reported by any experiment on humans and declared by any other authors.

\section{Data processing of DEGs}

R: The R Project for Statistical Computing(https://www.r-project.org/) was used to detect the DEGs between before inhalation(baseline sevoflurane) and after inhalation(sevoflurane) samples, and the adjusted P-value and $|\log F C|$ were calculated. We selected the $\mathrm{DEG}$ s by adjusting $\mathrm{P} \otimes 0.01$ and $|\log \mathrm{FC}| \geq 2.0$.

\section{GO and KEGG pathway analysis of DEGs}

$\mathrm{GO}$ analysis is a widely used method for functional enrichment studied. Also, gene functions were composed of biological process (BP), molecular function (MF), and cellular component (CC) three parts. KEGG is a large-scale used database, including vast amounts of genomes, biological pathways, diseases, chemicals, and drugs. We use the Database for Annotation, Visualization, and Integrated Discovery (DAVID) tools (https://david.ncifcrf.gov/) to analysis the DEGs through GO annotation analysis and KEGG pathway enrichment analysis. Once $P \llbracket 0.01$ and gene counts $\geq 10$, the genes were considered statistically significant. ${ }^{1}$

\section{PPI network construction and hub gene identification}

In this study, we use the Search Tool for the Retrieval of Interacting Genes (STRING) database (http://string-db.org/) and GeneMANIA online database (https://genemania.org/) to analyze the PPI information and evaluate the potential PPI relationship. They were also used to identify the DEGs to 
analysis the PPI information. A combined score was set to 0.4 , and then the PPI network was visualized by Cytoscape software (www.cytoscape.org/). The stability of the entire system was guaranteed by a higher degree node of connectivity. We calculated the degree of each protein node by using CytoHubba, a plugin in Cytoscape. Through those steps, we can select ten hub genes. ${ }^{1}$

\section{Results}

\section{Identification of DEGs}

Gene expression profile (GDS2772) was selected in this study using the following keywords: "sevoflurane," and "myocardial energy metabolism." GDS2772 contained 10 baseline sevoflurane samples, 10 sevoflurane samples. Based on the criteria of $\mathrm{P} \square 0.01$ and $|\log \mathrm{FC}| \geq 2$, a total of 726 DEGs were identified from baseline sevoflurane samples compared with sevoflurane samples, including 297 upregulated genes and 429 downregulated genes(Table $1)$. 

TICAM1 UTP4 FGFR1 C18965 IRF1 NR4A2 DNAJB1 AI939580 CHD1 MAPKAPK2 CHSY1 SLC39A14 ABL2 RELT MOB3C PPP1R15B INTS6 FOXK2 NFATC1 HES1 PRDM2 ATF3 H15073 MIR8085 XIRP1 ARL5B SRSF6 HBEGF

up-

\section{regulated}

429

\section{down-}

\section{regulated}

FOXC1 OTUD4 TIPARP AI540253 MAFK DUSP5 DUSP2 GPCPD1 SLC25A44 LRRC8A RASIP1 RASSF5 ATAD3B LOC100507258 FLNC ARID5B NFIL3 SLC1A5 STK40 NOP58 EGR1 RIPK2 SELE ARID5A COQ10B RARA-AS1 TRAPPC2B PIM1 ZBTB43 VAPA KLF6 THBD KLF4 RBBP6 AA824321 CDC42SE1 IER3 HIST2H2AA4 AKIRIN1 CEBPD JUND ZFP36 AI189587 STC1 EGR2 TCP11L1 EFNA1 LDLR KLHL21 DUSP14 SGK1 WARS CSF3 LDHA EIF2S1 NFKBIZ RAB20 SERTAD1 PHC2 MSANTD3 DUSP1 SPIDR BCAR1 GPRC5A SOX17 AI218358 MIDN FAM53C CD83 BAG3 HTRA3 PLEKHO2 IRS2 ZNF134 ISG20L2 PIM3 RND3 PNRC1 PDE4D TRIB1 RALGDS CENPN BCL6 VEGFA CSRNP1 TINF2 MYC NR4A3 PNPLA8 SOCS3 H2AFX LARP6 ATG101 SRSF3 TNFRSF12A EGR3 VPS37B ATF4 TNFAIP3 MCL1 CCL2 LOC101927933 NXT1 PSME4 CREM GMEB2 RPF2 GADD45B ADGRG1 KIAA0040 TNFAIP1 KCTD20 PPARD RGS2 SLC25A25 LINC00921 FOSL2 PPP1R15A DYRK3 NUP98 LATS2 AW057518 TUBB2A NIFK ZPR1 NAMPT NCS1 FOXC2 CCNL1 DUSP6 GRASP BRPF1 MAPK6 PXDC1 GPR183 GADD45A C17orf96 TMEM70 JMJD6 ADAMTS1 SLC7A5 PIGA KLHL15 CXCL2 GPR4 PAPD7 TWISTNB NKX3-1 NR4A1 TSC22D2 SERPINB1 DDX3Y TDG HSPA14 JUNB RHOB ZBTB21 APOLD1 METRNL EHD4 DNAJC2 MTRF1L FAM47E-STBD1 THBS1 ABL1 DNAJC27 CA2 IER5 TNFRSF10B SH2B3 RASD1 BHLHE40 WTAP MT1HL1 CDK2 PDE4B ERF CCNH PPRC1 HSPH1 FILIP1L MIR22 DNTTIP2 SOCS2 LOC101930489 ARC ACVR1B PRMT9 IL4R REL MAFG ZCCHC2 KBTBD2 PHF13 EHD1 CDKN1A NFKBIE SPSB1 ADAM17 USP36 FAT1 ARPC5L PTRH2 PTGR2 DLC1 ELMSAN1 ASXL1 ITPKC W91876 FOSB BCL6B C11orf96 ELL2 AK000203 NFKBIA LOC100996792 MIR24-2 LMNA BE467566 HES4 KLF1 0 NCOA7 LONRF1 ADNP2 B4GALT5 CSRNP2 GEM TFPI2 JUN MT2A EVA1C CRISPLD2 ATP1B3 SAMD4B ITPRIP BRAF CD274 CYR61 MAFF SIK1 TNFRSF10D FOSL1 NDEL1 AW137053 TP53BP2 CH25H SAT1 RYBP LOC100507507 SLC19A2 PVR PNP S1PR3 AF086069 RLF

COBLL1 ZNF225 RNF135 CNPY2 VAMP8 LMAN1 MDFIC LINC01279 EPB41L4B CRNKL1 MESDC2 SLC6A4 TXNDC15 POLI CHD9 POLG2 ITLN1 NINL CASP6 PPCS MIR6890 GTF2E1 FBXO8 AIDA STK26 TMEM60 ZNF623 STAG2 ANXA3 CYP2U1 EED SEL1L3 TRAF5 CALML4 WDR54 ALDH3A2 DDB2 RERG DPP4 AW972351 NPC2 NT5C1B-RDH14 WHSC1L1 GTF2IP4 AA588092 CACHD1 C3 C5orf15 PPP1R3D RIN2 CDKN1B POLR2B B3GNT7 GNPDA1 COMMD2 RARRES3 RFX3 BCHE SERPING1 BE044193 MEIS2 ARFGEF3 SKP2 SLC40A1 PHC3 C6orf203 METTL25 C12 orf75 DCAF12 C1 orf168 KBTBD6 C12 orf76 ALAD DHFR2 WDR19 PIGV EBLN3P EFHC1 EBPL MRGPRF RGL1 ANKRD49 TMEM14C ERMP1 ZNF641 NUDT16P1 NBR1 DCLRE1B SNORA11E TSN LZTFL1 SLC9A3R1 SMTNL2 EFNB3 IFIT2 NR3C1 ASTE1 DNALI1 HP PPL ZNF709 EXOC1 NBPF1 BICDL1 HPR CDK6 ZXDA FZD7 TBC1D2B IGSF9 SMG8 AMMECR1 WWC1 TTC30B TMEM14A UPK3B SYNRG LOC101929500 GATAD1 FAM160B1 SNCAIP MRFAP1L1 UPK1B SLC38A9 MAN1C1 DISP1 TCEANC PHGDH ZNF627 CBX1 SHPRH SLPI ZC3HAV1L ATP6V1A LOC646762 PRDM6 ERMARD VPS33B PARP4 IL18 GALNT12 RUNX1T1 SNX2 PRR15L ZFP90 SNUPN BMP4 CALB2 NAPEPLD HRASLS5 EPHB3 SNCA ITGB8 ZKSCAN4 BCAR3 SLC35B3 TRIM14 TRERF1 ZBTB7C TNFRSF8 MST1 RAB7B NIPAL2 FANCF KDELR3 FIGN LOC100288911 SLC35E2B SLC35A1 MAP3K1 ZBED8 PRRC1 LASP1 CBLN2 GPM6A CCDC28A PDIK1L AI307778 CA12 BE856302 DFNA5 ZBTB8A ZNF260 LINC00094 FBXL2 TLDC1 ZMAT3 PEX11B REEP1 ATF7IP NR2F2 LOC101930241 FAM84B ZBTB14 CREBL2 EFEMP1 BNC1 ATL1 COPG2IT1 C2CD5 CARHSP1 BLNK IFFO1 PDCD6 TMBIM4 KDR ZFP62 OGT BBS2 RBM4B ARHGAP32 KCNT2 INSIG2 IP6K2 CLDN15 IKZF2 MIR181A2HG EFNA5 DSC3 MN1 TTC31 IL17RD TBCC ERP29 LDLRAP1 PRG4 ARHGAP18 FAM8A1 UNC119B ZNF806 PRIM1 BACE1 HERPUD1 MYRF HACD3 JKAMP SMARCD2 CFI ZNF691 PALB2 CDON PTPRF GNAI1 VPS4B TRIM13 LOC102724562 ANXA8L1 ZBTB33 ARL4D TMEM203 NBPF10 FAM134A FADD FGF9 RPL1 5 ZNF844 NEO1 SRD5A1 ANKFY1 FIGNL1 TP53INP1 XPO1 CCDC80 H6PD SPOCK2 LINC01105 ABCC10 LPAR5 ELAC1 APITD1-CORT DENND6A TOM1L1 PRR15 PRKD1 PLPP6 NIF3L1 C21 orf62 RNASEL FAM69A ZDHHC23 AI469960 LRP2 PRSS23 CMTM4 C2 C5orf30 CNTNAP2 FRK ZNF616 C19orf33 PAPSS1 BTN3A3 KRT18 TRIM32 PKHD1L1 SMIM14 HTRA1 MPP5 WISP1 RTP4 EIF3L ADIRF LOC101928830 METTL7A LOC100506718 ARHGAP44 SSR1 SNORA70 UNC50 T89044 TMED10 SOX6 SKIDA1 AQP1 MBLAC2 LRRC1 ZFP64 GAS1 KRT19 RAB30 TMEM243 FLRT3 ABHD10 SEC11A LINC01133 TMEM128 TM7SF3 ZNF564 PLLP LOC101930363 MSLN SULF1 UBE2E2 SLC39A10 FNTA PCDHA1 APH1B RARRES1 SEMA4F QSER1 MBP ZNF286B TOR1AIP2 S100PBP SBSPON CFB PTPMT1 MAF FAM210B TET1 METTL13 IDH1 SPCS2 R33735 SHTN1 TBCK SMARCAD1 VSIG2 SMIM15 PCTP CLDN1 KIAA0430 COMMD3-BMI1 CGN FAM46C NSG1 CHRM3 ADNP DTWD1 ST3GAL5 AI683805 ZNF30 ASB7 FAM117B ZNF662 PTGS1 RABL2A FAM208A CYB5A CX3CR1 CLNS1A DBR1 FZD4 P2RX7 AA135722 PARD3B KLK11 PIGC BNC2 ANXA4 DCP1B ZKSCAN7 HOXB2 FKBP11 SLC4A4 ZNF133 RHOBTB1 AOX1 JRKL ZNF658 DAPK1 SELENBP1 PROCR PRKCA SLC18B1 SYT4 MAL2 LRP11 LRIG2 PARD6B TMEM37 SEMA3C TRMT5 ZNF420 TOB1 LOC101927204 WASF2 PTGIS KBTBD7 GOLPH3L BET1 AI683621 ZBED5 ZNF780A FNBP1 ZNF224 SLC35B4 ITPR3 SNORA60 


\section{Functional enrichment analyses of DEGs}

We analysis GO function and KEGG pathway enrichment analysis for DEGs using the DAVID (Table 2). The GO database was based on CC, BP, and MF ontologies. The results of the GO analysis indicated that DEGs were mainly involved in BPs and MFs. BPs, including positive regulation of endothelial cell migration, positive regulation of transcription from RNA polymerase II promoter and transcription, DNA-templated. MFs, including transcription factor activity, sequence-specific DNA binding, protein binding, sequencespecific DNA binding, and DNA binding. CC, including the nucleus. Besides, significantly enriched KEGG pathway of DEGs included PI3K-Akt signaling pathway, MAPK signaling pathway, Rap1 signaling pathway, TNF signaling pathway, and Neurotrophin signaling pathway and so on (Table 3). 


\begin{tabular}{|c|c|c|c|}
\hline Category & Term & Count & PValue \\
\hline GOTERM_BP_DIRECT & $\begin{array}{c}\text { GO:0010595 positive regulation of endothelial } \\
\text { cell migration }\end{array}$ & 11 & $\begin{array}{c}4.51 \mathrm{E}- \\
06\end{array}$ \\
\hline GOTERM_BP_DIRECT & $\begin{array}{l}\text { GO:0045944 positive regulation of } \\
\text { transcription from RNA polymerase II promoter }\end{array}$ & 64 & $\begin{array}{l}\text { 6.63E- } \\
06\end{array}$ \\
\hline \multirow[t]{2}{*}{ GOTERM_BP_DIRECT } & GO:0006351 transcription, DNA-templated & 108 & \\
\hline & & & $\begin{array}{l}6.86 \mathrm{E}- \\
06\end{array}$ \\
\hline \multirow[t]{2}{*}{ GOTERM_CC_DIRECT } & GO:0005634 nucleus & 241 & \\
\hline & & & $\begin{array}{c}2.78 \mathrm{E}- \\
06\end{array}$ \\
\hline GOTERM_MF_DIRECT & $\begin{array}{l}\text { GO:0003700 transcription factor activity, } \\
\text { sequence-specific DNA binding }\end{array}$ & 70 & $\begin{array}{l}3.22 \mathrm{E}- \\
08\end{array}$ \\
\hline \multirow[t]{2}{*}{ GOTERM_MF_DIRECT } & GO:0005515 protein binding & & \\
\hline & & 381 & $\begin{array}{l}9.94 \mathrm{E}- \\
08\end{array}$ \\
\hline \multirow[t]{2}{*}{ GOTERM_MF_DIRECT } & GO:0005515 protein binding & & \\
\hline & & 42 & $\begin{array}{l}2.17 \mathrm{E}- \\
06\end{array}$ \\
\hline \multirow[t]{2}{*}{ GOTERM_MF_DIRECT } & GO:0003677 DNA binding & & \\
\hline & & 93 & $\begin{array}{l}2.48 \mathrm{E}- \\
05\end{array}$ \\
\hline
\end{tabular}

Table 2 GO function enrichment analysis of DEG 


\begin{tabular}{ccc}
\hline Term & Count & PValue \\
\hline hsa05161:Hepatitis B & 16 & $3.85 E-04$ \\
hsa05200:Pathways in cancer & 27 & 0.003972827 \\
hsa05219:Bladder cancer & 7 & 0.004221366 \\
hsa05164:Influenza A & 15 & 0.006402674 \\
hsa04668:TNF signaling pathway & 11 & 0.007356759 \\
hsa04010:MAPK signaling pathway & 19 & 0.007532121 \\
hsa04115:p53 signaling pathway & 8 & 0.013279295 \\
hsa04015:Rap1 signaling pathway & 16 & 0.013754862 \\
hsa04012:ErbB signaling pathway & 9 & 0.017306612 \\
hsa04110:Cell cycle & 11 & 0.01949773 \\
hsa04151:PI3K-Akt signaling pathway & 22 & 0.022026509 \\
hsa05168:Herpes simplex infection & 14 & 0.022130925 \\
hsa05133:Pertussis & 8 & 0.02348344 \\
hsa05203:Viral carcinogenesis & 15 & 0.02407624 \\
hsa05160:Hepatitis C & 11 & 0.030008383 \\
hsa05166:HTLV-I infection & 17 & 0.032283344 \\
hat72:Neurotrophin signaling pathway & 10 & 0.038971746 \\
hsa05205:Proteoglycans in cancer & 14 & 0.041273736 \\
hsa05169:Epstein-Barr virus infection & 10 & 0.042608302 \\
hsa05215:Prostate cancer & 8 & 0.049777894 \\
\hline
\end{tabular}

Table 3 KEGG pathway enrichment analysis of DEG

\section{PPI network construction and hub gene identification}

PPI network analysis among the DEGs was predicted with STRING tools and GeneMANIA online database. The STRING online database and GeneMANIA online database were performed to analyse the protein-protein interaction networks of the differentially expressed genes and modular, as presented in Figure 1. Those 10 genes showed by connectivity degree in the PPI network were identified (Figure 2). The results showed that Jun proto-oncogene, AP-1 transcription factor subunit(JUN) was the most outstanding gene with connectivity degree=93, followed by baculoviral early growth response 1 (EGR1; degree=55), activating transcription factor 3 (ATF3; degree=48), FosB proto-oncogene, AP-1 transcription factor subunit (FOSB; degree=34), JunB proto-oncogene, AP-1 transcription factor subunit (JUNB; degree=28), dual specificity phosphatase 1(DUSP1;degree=39), early growth response 2 (EGR2; degree=26), nuclear receptor subfamily 4 group A member 1 (NR4A1; degree=32), BTG anti-proliferation factor 2 
(BTG2; degree=26), nuclear receptor subfamily 4 group A member 2 (NR4A2; degree=25). All of these hub genes were upregulated after sevoflurane inhalation.

\section{Discussion}

We have already known sevoflurane has myocardial protective effects. DEGs between before inhalation (baseline sevoflurane) and after inhalation (sevoflurane) samples based on gene expression profiling data were screened out from the GEO database. Finally, we identified 297 upregulated DEGs and 429 downregulated DEGs. The GO annotation analysis of those selected DEGs shows that BP terms including positive regulation of endothelial cell migration, positive regulation of transcription from RNA polymerase II promoter and transcription, DNA-templated, MFs terms including transcription factor activity, sequencespecific DNA binding, protein binding, sequence-specific DNA binding and DNA binding and CCs including the nucleus. KEGG pathway analysis showed that DEGs were mainly enriched in pathways in the PI3K-Akt signaling pathway, MAPK signaling pathway, Rap1 signaling pathway, TNF signaling pathway, and Neurotrophin signaling pathway, and so on. A PPI network was constructed to investigate the interrelationship of the DEGs, and ten hub genes were identified, including JUN, EGR1, ATF3, FOSB, JUNB, DUSP1, EGR2, NR4A1, BTG2, NR4A2.

EGR1 encodes the protein which belongs to the EGR family of $\mathrm{C} 2 \mathrm{H} 2$-type zinc-finger proteins. The protein is a nuclear protein that performs transcription. The products of target genes are necessary for differentiation and mitogenesis.9 Xiang Y, et al. find that after undergoing Percutaneous Transluminal Coronary Intervention(PCI) in CHD patients, once EGR1 level significantly decreased once in the early postoperative period, the patient's coronary may be suspected not having reflow and should be examined timely to improve the effectiveness of therapeutic. 10 EGR1 has been named for the widespread presence and expressing rapidly in human cells. Shajahan-Haq AN, et al. showing that EGR1 has a complex signal pathway, playing a significant role in cell growth and affecting differentiation, proliferation, and inflammatory response.11 Another article, once in the plasma of patients with coronary heart disease, the expression level of EGR1 decreased, it suggests the aggravating of the disease of patients. The level of EGR1can be used to assess the patient's current status and the progression of disease.12

ATF3 is a gene encoding transcription factors of the mammalian activation transcription factor/cAMP responsive element-binding (CREB) protein family. This gene is induced by a variety of signals, which is related to the happening of the cancer cells and the complex process of the cellular responding to stress. This gene has multiple transcript variants encoding different isoforms. Alternative splicing of this gene may play an important physiological role in regulating target genes. $\mathrm{Li} \mathrm{YU}$, et al. shows that upregulation of ATF3 can reduce the heart damage and heart failure induced by hypertension and inhibit cardiac fibroblast cells.13

FOS encodes proteins having been implicated significant relationship with cell proliferation, differentiation, and transformation. The Fos gene family consists of 4 members, including FOS, FOSB, FOSL1, and FOSL2. Dunand-Sauthier I,et al find that c-Fos participates in proliferation, differentiation and 
apoptosis various cellular processes. ${ }^{6}$ Several studies have shown that c-Fos decreases proinflammatory cytokine production, and decrease cardiomyocytes death under conditions of hypoxia. ${ }^{7}$

The protein encoded by BTG2 is a member of the BTG/Tob family which has structurally related proteins have antiproliferative function and is involved in the regulations of the G1/S transition during the cell cycle. BTG2 only affect cytosolic, but not nuclear, RNA levels. Masumura Y, et al shows that under adrenergic stimulation, BTG2 knockdown further enhances cytosolic RNA accumulation in cardiomyocytes. It indicates that BTG2 decrease RNA accumulation to protect cardiomyocytes from hypertrophy. ${ }^{5}$

The protein encoded by JUNB is a member of the activator protein-1 (AP-1) transcription factor family including Jun (c-Jun, JunB and JunD) and Fos (c-Fos, FosB, Fra-1 and Fra-2). ${ }^{14}$ JunB combines with the cognitive binding sequence localized in the cis-regulatory region of target genes and participates in cell cycle, proliferation, and apoptosis biological processes. Szremska AP,et al. and Gurzov EN, et al. find that overexpressing JunB can inhibit the proliferation of malignant keratinocytes in mouse, ${ }^{15}$ and inhibit cell apoptosis in pancreatic beta cells. ${ }^{1618}$ Also, Chen J, et al. find that JunB can protect heart failure区HF区 from inflammatory cardiomyopathy, ${ }^{19}$ while in zebrafish decreased JunB leads to HF. ${ }^{20}$

The protein encoded by DUSP1 is an anti-apoptotic phosphatase, and exited in a wide variety of organizations, especially having a high level in the heart. ${ }^{21}$ One study finds that once I/R injuries happening, decreased DUSP1 can lead to scar expansion, cardiac dysfunction, and cellular death. The absence of DUSP1 triggered fatal mitochondrial fission leading to extensive cell death through increased JNK phosphorylation and the expression of Mff. ${ }^{22}$

NR4A1 encodes a member of the steroid-thyroid hormone-retinoid receptor superfamily. The encoded protein acts as a nuclear transcription factor. When the protein encoded by NR4A1 transferred from nucleus to the mitochondria leads to apoptosis. It has different subtypes of several transcriptional variants which have an efficient function of cardiac repair. ${ }^{23}$

The protein encoded by BTG2 is affiliated to the BTG/Tob family. This family related proteins have antiproliferative properties. The protein encoded by BTG2 plays an important role in regulating the $\mathrm{G} 1 / \mathrm{S}$ transition of the cell cycle. BTG2 can impact the accumulation of cytosolic, but not nuclear, RNA levels. Masumura $\mathrm{Y}$, et al. suggest that though down-regulating the cardiomyocytes accumulation of RNA, BTG2 can attenuate reactive hypertrophy. ${ }^{5}$

NR4A2, nuclear receptor subfamily 4, group A, member 2, with NR4A1 and NR4A3 composed NR4A orphan nuclear receptor family. ${ }^{24}$ The NR4A family is the immediate early response transcription factors, which has great relationship with ischemic stroke. ${ }^{25}$ NR4A2 participates the apoptosis of various cancer cells under stress through different functions. ${ }^{26}$ In the MI injury mouse, NR4A2 is upregulated. Furthermore, NR4A2 can protect heart from apoptosis and hypertrophy, and also alleviate heart injury. 


\section{Conclusions}

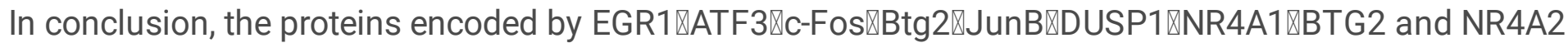
were related to cardiac function. ATF3, FOSB, JUNB, DUSP1, NR4A1, NR4A2 are related to apoptosis of cardiomyocytes. The protein encoded by BTG2 is related to hypertrophy. Sevoflurane regulates cell transcription, inflammatory and apoptosis through those hub genes to protect myocardial.

\section{Abbreviations}

OPCABG: Off-pump coronary artery bypass graft; GEO: Gene Expression Omnibus; GO: Gene Ontology; KEGG: Kyoto Encyclopedia of Genes and Genomes; DEGs: Differentially expressed genes; PPI: Proteinprotein interaction; CAD: Coronary artery disease; PCl: Percutaneous Transluminal Coronary Intervention; CREB: CAMP responsive element-binding; AP-1: Activator protein-1; ER: Endoplasmic reticulum; HF: Heart failure; CMT1D: Charcot-Marie-Tooth disease type 1D; CMT4E: Charcot-Marie-Tooth disease type 4E; DSS: Dejerine-Sottas syndrome.

\section{Declarations}

\section{Ethics approval and consent to participate}

Not applicable.

\section{Consent for publication}

Not applicable.

\section{Availability of data and materials}

The datasets used and analysed during the current study are available from the corresponding author upon reasonable request.

\section{Competing interests}

The authors declare that they have no competing interests.

\section{Funding}

There was no external funding. Support was provided solely from institutional and/or departmental sources.

\section{Authors' contributions}

Hua Lin analyzed and interpreted the data and was a major contributor in writing the manuscript. All authors read and approved the final manuscript. 
We thank Dr. Liu Hongwei for his helpful comments.

\section{References}

1. Bu X, Wang B, Wang Y, Wang Z, Gong C, Qi F, Zhang C. Pathway-related modules involved in the application of sevoflurane or propofol in off-pump coronary artery bypass graft. Exp Ther Med. 2017;14:97-106. doi: 10.3892/etm.2017.4504.

2. Zhu P, Chen A, Wang Z, Ye X, Zhou M, Liu J, Zhao Q. Long-term outcomes of multiple and single arterial off-pump coronary artery bypass grafting. J Thorac Dis.2019;11:909-919. doi: 10.21037/jtd.2019.01.101.

3. Wan, H, Lyu Y, Liao Q, Jin L, Xu L, Hu Y, Yu Y, Guo K. Effects of Remote Ischemic Preconditioning in Patients Undergoing Off-Pump Coronary Artery Bypass Graft Surgery. Front Physiol.2019; 29(10):495. doi: 10.3389/fphys.2019.00495.

4. Zhou C, Martinez E, Di Marcantonio D, Solanki-Patel N, Aghayev T, Peri S, Ferrar, F, Skorski T, Scholl C, Fröhling S, Balachandran S, Wiest DL, Sykes SM. JUN is a key transcriptional regulator of the unfolded protein response in acute myeloid leukemia. 2017; 31:1196-1205. doi: 10.1038/leu.2016.329.

5. Masumura Y,Higo S, Asano Y, Kato H, Yan Y, Ishino S, Tsukamoto O, Kioka H, Hayashi T, Shintani Y Yamazaki S, Minamino T, Kitakaze M, Komuro I, Takashima S, Sakata Y. Btg2 is a Negative Regulator of Cardiomyocyte Hypertrophy through a Decrease in Cytosolic RNA. Sci Rep. 2016;27 (6):28592. doi: 10.1038/srep28592.

6. Dunand-Sauthier I, Santiago-Raber ML, Capponi L, Vejnar CE Schaad O, Irla M, Seguín-Estévez Q, Descombes P, Zdobnov EM, Acha-Orbea H, Reith W. Silencing of c-Fos expression by microRNA155 is critical for dendritic cell maturation and function. 2011;117:4490-500. doi: 10.1182/blood2010-09-308064.

7. Zhu J, Yao K, Guo J, Shi H, Ma L, Wang Q, Liu H, Gao W, Sun A, Zou Y, Ge J. miR-181a and miR-150 regulate dendritic cell immune inflammatory responses and cardiomyocyte apoptosis via targeting JAK1-STAT1/c-Fos pathway. J Cell Mol Med.2017;21(11): 2884-2895. doi: 10.1111/jcmm.13201.

8. Villa Del Campo C, Lioux G, Carmona R, Sierra R, Muñoz-Chápuli R, Clavería C, Torres M. Myc overexpression enhances of epicardial contribution to the developing heart and promotes extensive expansion of the cardiomyocyte population. Sci Rep. 2016; 18(6):35366. doi: 10.1038/srep35366.

9. Duclot $F$ and Kabbaj M. The role of early growth response 1 (EGR1) in brain plasticity and neuropsychiatric disorders. Front Behav Neurosci. 2017;6(11):35.doi: 10.3389/fnbeh.2017.00035.

10. Xiang Y, Peng J, Liu Q, Guo C. The association between CD69 and EGR1 levels, and CHD patients without reflow after PCI. Exp Ther Med.2019;17(5):3913-3920. doi: 10.3892/etm.2019.7411.

11. Shajahan-Haq AN, Boca SM, Jin L, Bhuvaneshwar K, Gusev Y, Cheema AK, Demas DD, Raghavan KS, Michalek R, Madhavan S, Clarke R. EGR1 regulates cellular metabolism and survival in endocrine 
resistant breast cancer. 2017;8:96865-96884. doi: 10.18632/oncotarget.18292.

12. Peng J, Xiang Y. Value analysis of CD69 combined with EGR1 in the diagnosis of coronary heart disease. Exp Ther Med.2019; 17(3):2047-2052. doi: 10.3892/etm.2019.7175.

13. Li Y, Li Z, Zhang C, Li P, Wu Y, Wang C, Bond Lau W, Ma XL, Du J. Cardiac Fibroblast-Specific Activating Transcription Factor 3 Protects Against Heart Failure by Suppressing MAP2K3-p38 Signaling. 2017;135:2041-2057. doi: 10.1161/CIRCULATIONAHA.116.024599.

14. Papoudou-Bai A, Goussia A, Batistatou A, Stefanou D, Malamou-Mitsi V, Kanavaros P. The expression levels of JunB, JunD and p-c-Jun are positively correlated with tumor cell proliferation in difuse large B-cell lymphomas. Leuk Lymphoma. 2016;57:143-50. doi: 10.3109/10428194.2015.1034704.

15. Szremska AP, Kenner L, Weisz E, Ott RG, Passegué E, Artwohl M, Freissmuth $M$, Stoxreiter R, Theussl $H C$, Parzer SB, Moriggl R, Wagner EF, Sexl V. JunB inhibits proliferation and transformation in Blymphoid cells. 2003; 102(12):4159-65.

16. Gurzov EN, Ortis F, Bakiri L, Wagner EF, Eizirik DL. JunB Inhibits ER Stress and Apoptosis in Pancreatic Beta Cells. PLoS One.2008;3:e3030. doi: 10.1371/journal.pone.0003030.

17. Kiesow K, Bennewitz K, Miranda LG, Stoll SJ, Hartenstein B, Angel P, Kroll J, Schorpp-Kistner M. Junb controls lymphatic vascular development in zebrafsh via miR-182. Sci Rep.2015;5:15007. doi: 10.1038/srep15007.

18. Licht AH, Pein OT, Florin L, Hartenstein B, Reuter H, Arnold B, Lichter P, Angel P, Schorpp-Kistner M. JunB is required for endothelial cell morphogenesis by regulating core-binding factor beta. $\mathrm{J}$ Cell Biol.2006; 175:981-91.

19. Chen J, Wang HY, Zeng CY. Transcriptome network analysis of potential candidate genes for heart failure. Genet Mol Res.2013;12:4687-97. doi: 10.4238/2013.

20. Meder B, Just S, Vogel B, Rudloff J, Gärtner L, Dahme T, Huttner I, Zankl A, Katus HA, Rottbauer W. JunB-CBFbeta signaling is essential to maintain sarcomeric Z-disc structure and when defective leads to heart failure. J Cell Sci. 2010; 123(15):2613-20. doi: 10.1242/jcs.067967.

21. Kwak SP, Hakes DJ, Martell KJ, Dixon JE. Dixon. Isolation and characterization of a human dual specificity protein-tyrosine phosphatase gene. J Biol Chem. 1994;269:3596-604.

22. Jin Q, Li R, Hu N, Xin T, Zhu P, Hu S, Ma S, Zhu H, Ren J, Zhou H. DUSP1 alleviates cardiac ischemia/reperfusion injury by suppressing the Mff-required mitochondrial fission and Bnip3-related mitophagy via the JNK pathways. Redox Biol.2018;14:576-587. doi: 10.1016/j.redox.2017.11.004.

23. Dehn S, Thorp EB. Myeloid receptor CD36 is required for early phagocytosis of myocardial infarcts and induction of Nr4a1-dependent mechanisms of cardiac repair. FASEB J.2018;32:254-264. doi: 10.1096/fj.201700450R.

24. Volakakis N, Malewicz M, Kadkhodai B, Perlmann T, Benoit G. Characterization of the Nurr1 ligandbinding domain co-activator interaction surface. J Mol Endocrinol.2006;37:317-26.

25. Xiao G, Sun T, Songming C, Cao Y. NR4A1 enhances neural survival following oxygen and glucose deprivation: an in vitro study. J Neurol Sci.2013;330:78-84. doi: 10.1016/j.jns.2013.04.010. 
26. Beard JA, Tenga A, Chen T. The interplay of NR4A receptors and the oncogene-tumor suppressor networks in cancer. Cell Signal.2015;27:257-66. doi: 10.1016/j.cellsig.2014.11.009.

\section{Figures}

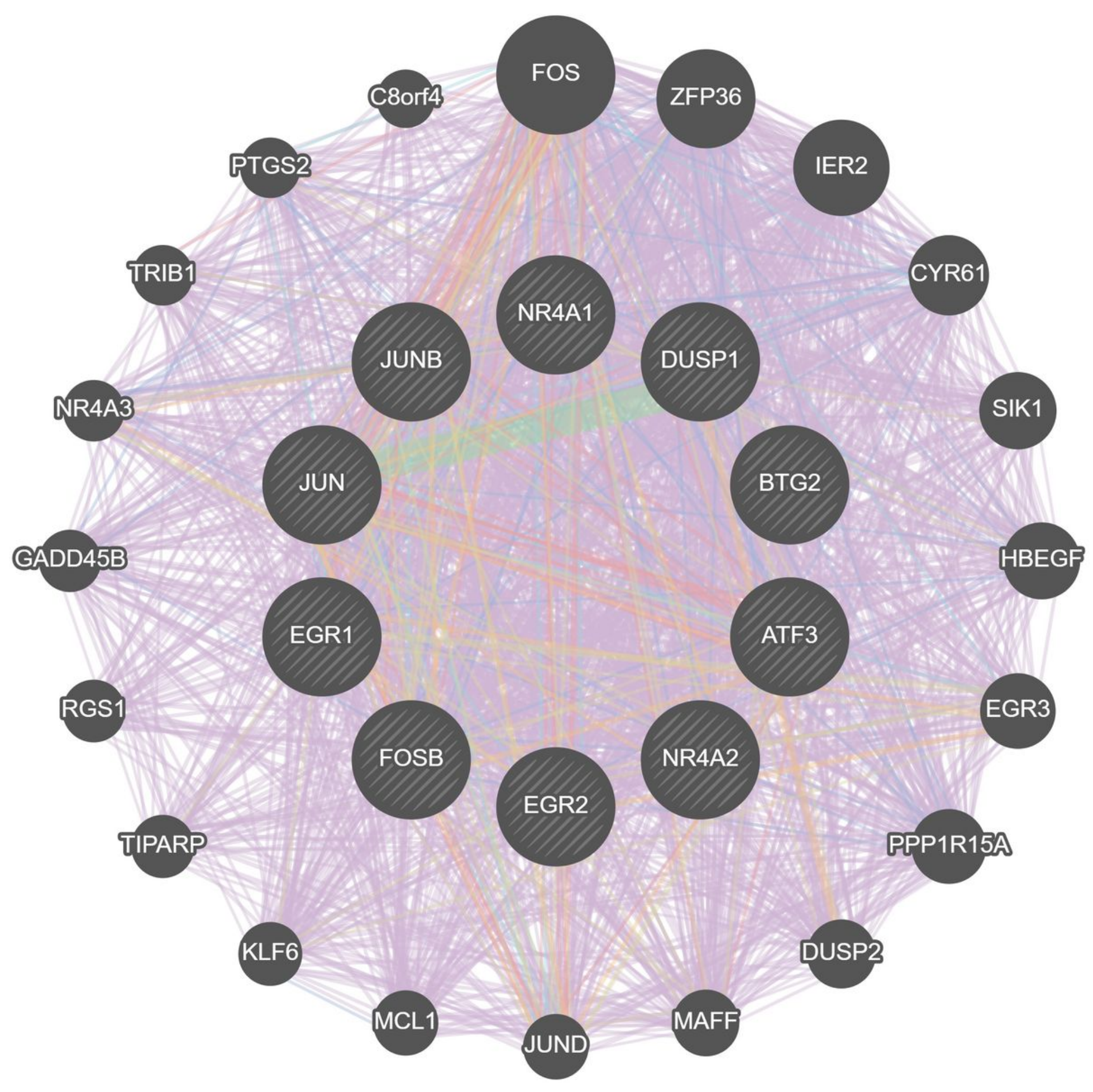

\section{Figure 1}

Genemania network 


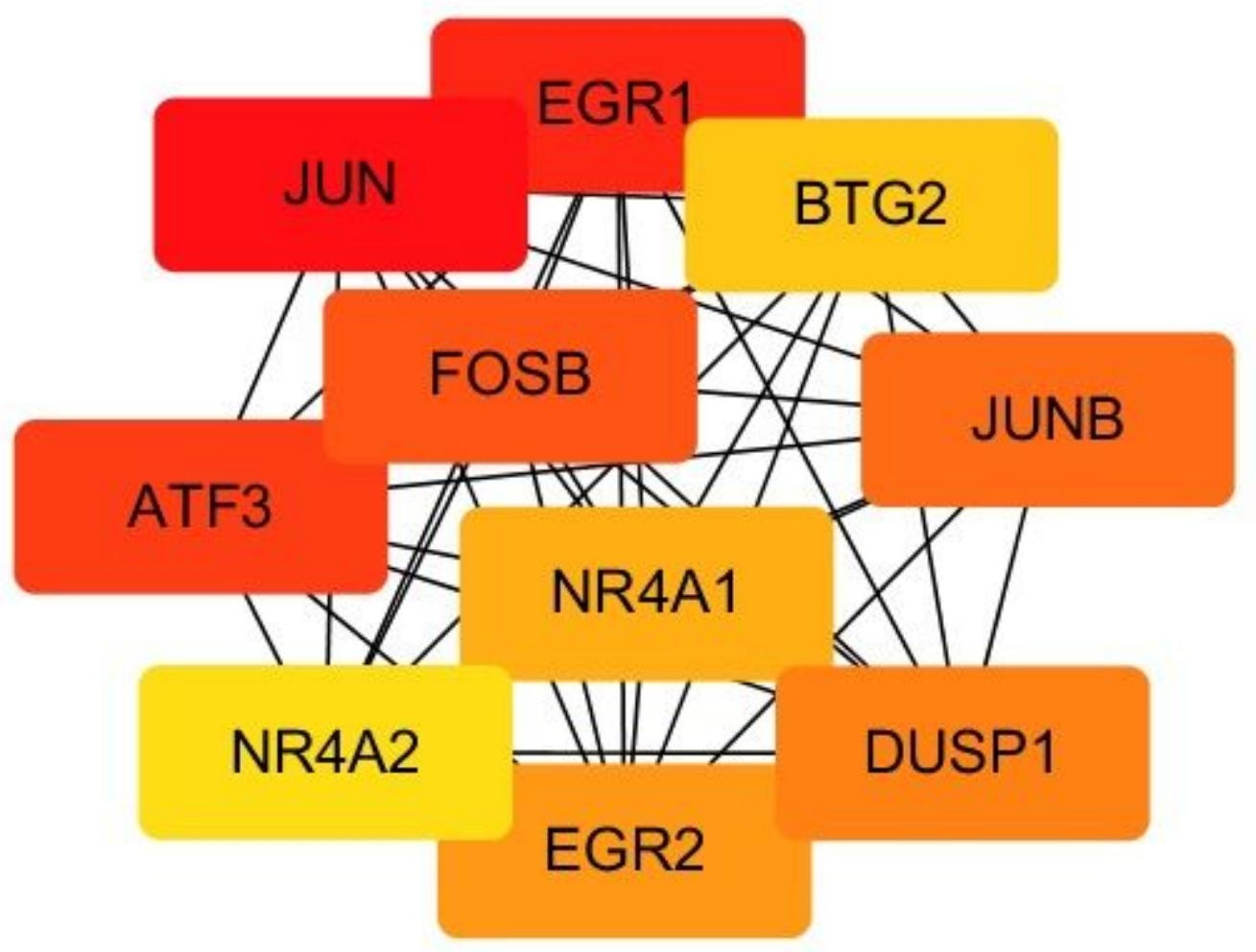

Figure 2

Ten hub genes 